\title{
Non-selective Beta-Adrenergic Agonist Infusion Acutely Stimulates the Temperature of Brown Adipose Tissue in Adult Males
}

H Scotney ${ }^{1}$, M Symonds ${ }^{1}$, J Law ${ }^{1}$, H Budge ${ }^{1}$, D Sharkey ${ }^{1}$ and K Manolopoulos ${ }^{2}$

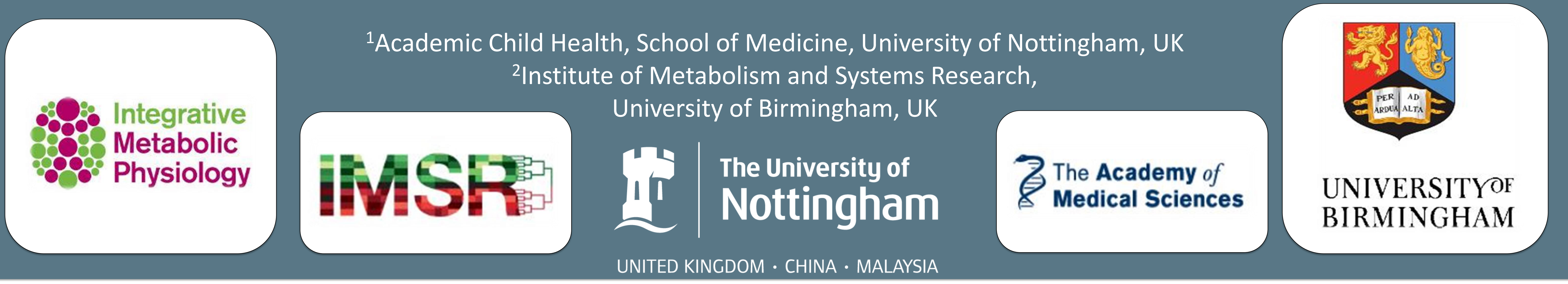

\section{Background}

Over the past decade, brown adipose tissue (BAT) has emerged as a potential therapeutic target to combat childhood and adult obesity, due to its potential to dissipate energy through mitochondrial uncoupling.

There is convincing evidence to suggest that BAT is under sympathetic control and can be activated through $\beta$ adrenoceptor (AR) stimulation. Hypercortisolism, when pharmacologically induced in rodents, leads to an inhibitory effect on BAT activity and suppression of norepinephrine-induced mitochondrial uncoupling. Little is known about the role of glucocorticoids in human BAT function, and whether they affect adrenergic activation of thermogenesis.

\section{Aims}

1. To examine whether nonselective ß-AR stimulation with Isoprenaline (ISO) activates BAT in humans, measured by Infrared Thermography (IT).

2. To investigate the effects of acute hypercortisolaemia on human BAT function by infusing hydrocortisone (HC).

\section{Methods}

Eight healthy males (Table 1 ) were studied following $\mathrm{HC}$ infusion (0.2 $\mathrm{mg} . \mathrm{kg}^{-1} \cdot \mathrm{min}^{-1}$ for $16 \mathrm{hrs}$ ) or saline. Infusions were given in a double-blind, randomized fashion. BAT thermogenesis was measured by IT before, during, and after a 60 min ISO infusion (25 ng.kg fat free mass $^{-1} \cdot \mathrm{min}^{-1}$ ). Thermal images were taken at a rate of $4 / \mathrm{min} 15 \mathrm{~min}$ prior to and throughout the ISO infusion, and $2 / \mathrm{min}$ for $15 \mathrm{~min}$ post infusion. The temperature of the supraclavicular fossa was analysed using Matlab software (Figure 1). iButtons recorded skin temperature every minute and were taped within the supraclavicular fossa (main site of BAT) and lateral of the umbilicus. $\mathrm{HR}$ and $\mathrm{BP}$ were recorded every 5 minutes using continuous ECG monitoring. ANOVA' statistics were used with $P<0.05$. Ethical approval was given.

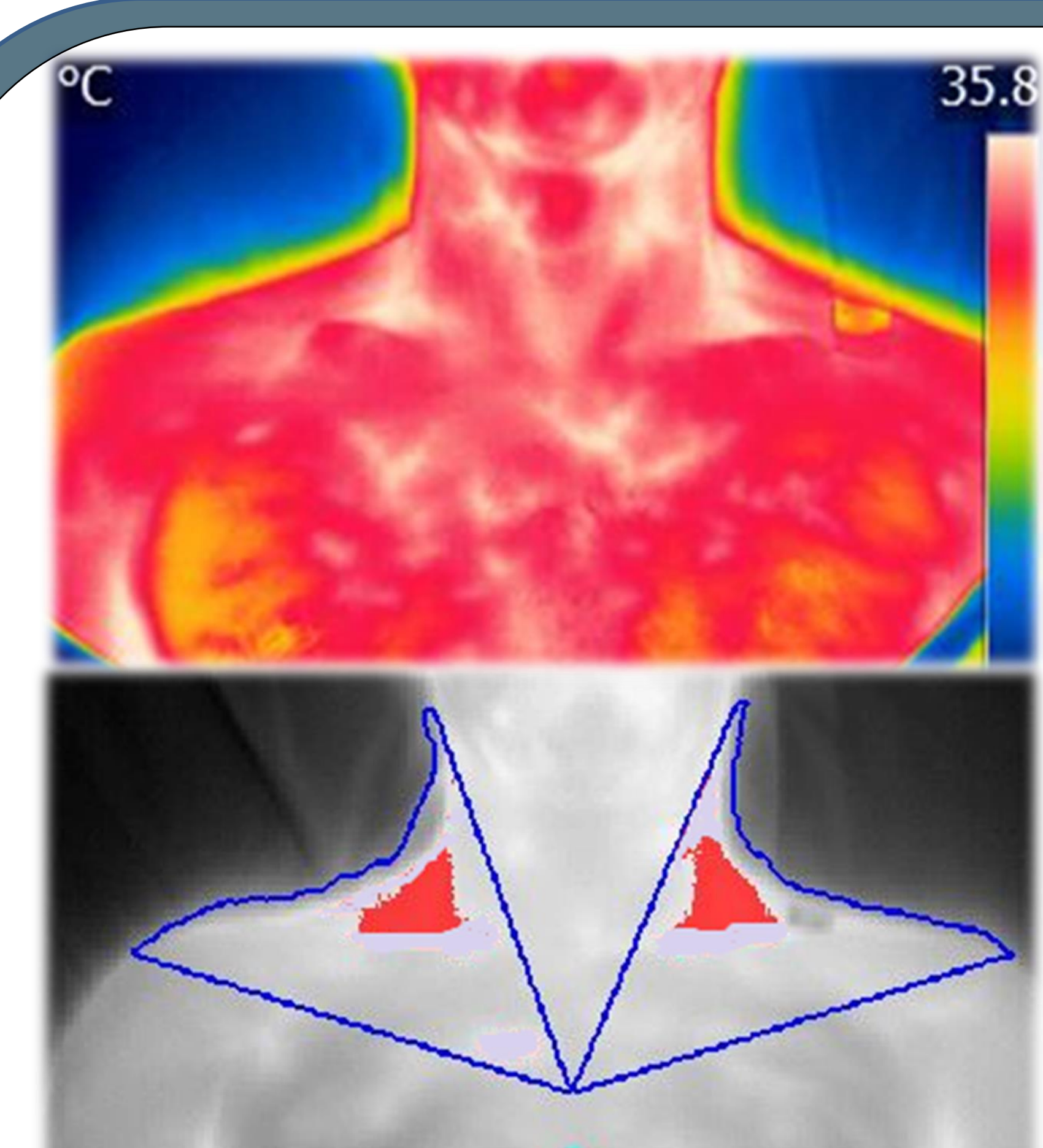

Table 1. Participant characteristics
\begin{tabular}{|l|l|}
\hline N & $\mathbf{8}$ males \\
\hline Age (years) & $23.13 \pm 4.53$ \\
\hline Weight $(\mathbf{k g})$ & $74.13 \pm 6.92$ \\
\hline BMI $\left(\mathbf{k g} \cdot \mathbf{m}^{2}\right)$ & $22.78 \pm 1.12$ \\
\hline WHR & $0.84 \pm 0.05$ \\
\hline Trunk Fat (g) & $7538 \pm 1412$ \\
\hline Leg Fat (g) & $5580 \pm 961$ \\
\hline Visceral Fat (g) & $202 \pm 96$ \\
\hline
\end{tabular}

Mean \pm Standard deviation shown; NEFA, non-esterified fatty acids Fat mass measurements obtained by DXA

\section{Results}

ISO infusion resulted in significant ß-adrenergic mediated increases in heart rate $(47 \pm 4 \mathrm{bpm}$ ) peaking at $45 \mathrm{~min}$ (Figure 2a).

ISO infusion resulted in a highly localized increase in local temperature within the supraclavicular region compared to baseline $\left(\Delta 0.54 \pm 0.08^{\circ} \mathrm{C}, P=0.001\right.$ [Figure $2 \mathrm{c}, \mathrm{d}$ ] $)$

$\mathrm{HC}$ resulted in a lesser increase in BAT temperature during ISO infusion $\left(\triangle 0.51 \pm 0.09^{\circ} \mathrm{C}, P=0.01\right.$ [Figure $2 \mathrm{c}, \mathrm{d}$ ]). Further, basal BAT temperature is increased in $\mathrm{HC}$ compared to saline (Figure 2b).

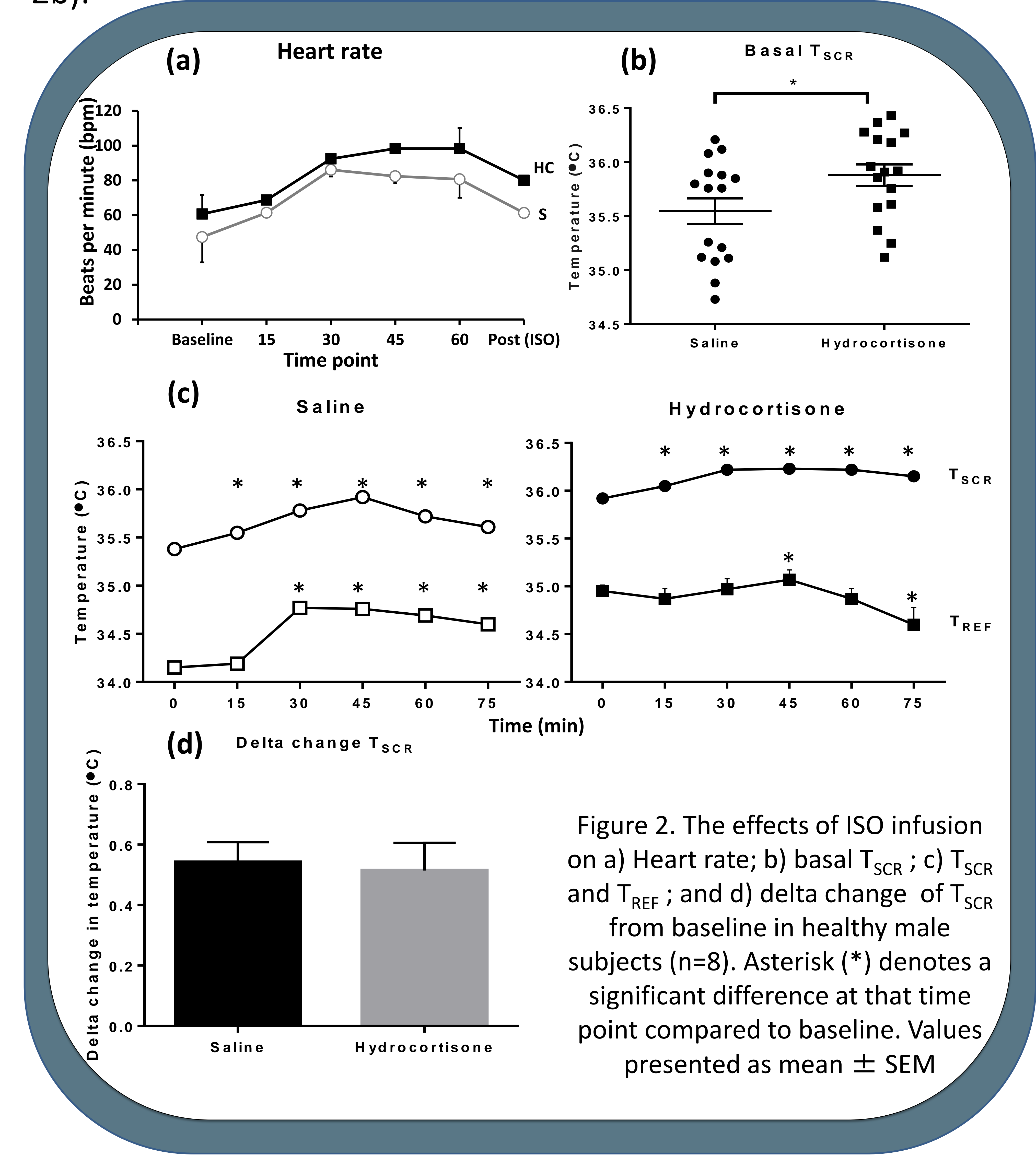

\section{Conclusions}

ß-adrenergic stimulation induced dynamic changes in BAT activity that was measurable by IT. This confirms IT as a simple, noninvasive method for assessment of real-time BAT function. Acute hypercortisolaemia resulted in blunting of the adrenergicmediated increase in BAT activity, possibly due to glucocorticoiddependent BAT pre-activation. Future studies should focus on the effects of chronic glucocorticoid excess on human BAT function, and whether tissue-specific inhibition of glucocorticoid activity/generation can contribute to increased BAT thermogenesis. 